\title{
SEPARATE HOLOMORPHIC EXTENSION ALONG LINES AND HOLOMORPHIC EXTENSION FROM THE SPHERE TO THE BALL
}

\author{
LUCA BARACCO
}

\begin{abstract}
We give positive answer to a conjecture by Agranovsky. A continuous function on the sphere which has separate holomorphic extension along the complex lines which pass through three non aligned interior points, is the trace of a holomorphic function in the ball.

MSC: 32F10, 32F $20,32 \mathrm{~N} 15,32 \mathrm{~T} 25$
\end{abstract}

\section{INTRODUCTION}

The problem of describing families of discs which suffice for testing analytic extension of a function $f$ from the sphere $\partial \mathbb{B}^{2}$ to the ball $\mathbb{B}^{2}$ has a long history. For $f$ continuous on $\partial \mathbb{B}^{2}$, Agranovsky-Valski [4] use all the lines, Agranovki-Semenov [3] the lines through an open subset $D^{\prime} \subset \mathbb{B}^{2}$, Rudin [10] the lines tangent to a concentric subsphere $B_{\frac{1}{2}}^{2}$, Baracco-Tumanov-Zampieri the lines tangent to any strictly convex subset $D^{\prime} \subset \subset \mathbb{B}^{2}$. There are many other contributions such as [2], [11], [8] just to mention a few. It is a challenging attempt to reduce the number of parameters in the testing families. However, one encounters an immediate constraint: lines which meet a single point $z_{o} \in \mathbb{B}^{2}$ do not suffice. Instead, two interior points or a single boundary point suffice: Agranovsky [1] and Baracco [5]. However, in these last two results, the reduction of the testing families is compensated by an assumption of extra initial regularity: $f$ is assumed to be real analytic. Globevnik [7] shows that, for two points, $C^{\infty}$-regularity still suffices, but $C^{k}$ does not. This suggests that holomorphic extension is a good balance between reduction of testing families and improvement of initial regularity. And in fact, it is showed here, that for $f \in C^{0}$ three not on the same line points suffice. Here is our result.

Theorem 1.1. Let $f$ be a continuous function on the sphere $\partial \mathbb{B}^{2}$ which extends holomorphically along any complex line in $\mathbb{B}^{2}$ which encounters the set consisting of 3 points not on the same line. Then, $f$ extends holomorphically to $\mathbb{B}^{2}$. 
The proof follows in Section 2 below. It shows that, the result should hold for a ball of general dimension $\mathbb{B}^{n}$. In this case, $n+1$ points in generic position should suffice. We first introduce some terminology. A straight disc $A$ is the intersection of a straight complex line with $\mathbb{B}^{2} ; \mathbb{P} T^{*} \mathbb{C}^{2}$ is the cotangent bundle with projectivized fibers, and $\pi$ the projection on the base space; $\mathbb{P} T_{\partial \mathbb{B}^{2}}^{*} \mathbb{C}^{2}$ the projectivized conormal bundle to $\partial \mathbb{B}^{2}$ in $\mathbb{C}^{2}$. It is readily seen that the straight discs $A$ of the ball are the geodesics of the Kobayashi metric, or, equivalently, the so called "stationary discs" (cf. Lempert [9]). These are the discs endowed with a meromorphic lift $A^{*} \subset \mathbb{P} T^{*} \mathbb{C}^{2}$ with a simple pole attached to $T_{\partial \mathbb{B}^{2}}^{*} \mathbb{C}^{2}$, that is, satisfying $\partial A^{*} \subset \mathbb{P} T_{\partial \mathbb{B}^{2}}^{*} \mathbb{C}^{2}$. We fix three points $P_{j}$, $j=1,2,3$ in $\mathbb{B}^{2}$ and consider a set, indexed by $j$, of (2)-parameter families of straight discs $A^{j}$ passing through $P_{j}$. We define $M_{j}$ to be the union of the lifts of the family with index $j$. The set $M_{j}$ is generically a CR manifold with $\mathrm{CR}$ dimension 1 except at the points that project over $P_{j}$; we denote by $M_{j}^{\text {reg }}$ the complement of this set. The boundary of $M_{j}$ concides with $\mathbb{P} T_{\partial \mathbb{B}^{2}}^{*} \mathbb{C}^{2}$ which is maximal totally real in $\mathbb{P} T^{*} \mathbb{C}^{2}$. Here is the central point of our construction. Though the function $f$, in the beginning of the proof, is not extendible to $\mathbb{B}^{2}$ as a result of the separate extensions to the $A$ 's, nevertheless it is naturally lifted to a function $F$ on $M_{j}$ by gluying the bunch of separate holomorphic extensions to the lifts $A^{*}$ 's. This is defined by

$$
F(z,[\zeta])=f_{A_{(z,[\zeta])}}(z),
$$

where $A_{(z,[\zeta])}$ is the unique stationary disc whose lift $A_{(z,[\zeta])}^{*}$ passes through $(z,[\zeta])$. The crucial point here is that the $A$ 's may overlap on $\mathbb{C}^{2}$ but the $A^{*}$ 's do not in $\mathbb{P} T^{*} \mathbb{C}^{2}$. The function $F$ is therefore well defined and $\mathrm{CR}$ on $M_{j}^{\mathrm{reg}}$.

\section{Proof of Theorem 1.1}

The proof consists of several steps. We start by collecting some easy computations. We identify $\mathbb{P} T^{*} \mathbb{C}^{2} \simeq \mathbb{C}^{2} \times \mathbb{C P}_{1} \simeq \mathbb{C}^{3}$ with coordinates $\left(z_{1}, z_{2}\right) \in \mathbb{C}^{2}$ and $z_{3}=\frac{\zeta_{2}}{\zeta 1} \in \mathbb{C P}_{1}$. Let $M_{0}$ be the collection of the lifts of the discs through 0 .

Lemma 2.1. Let $A_{0}^{*}$ be the (unique) disc of $M_{0}$ which projects over the $z_{1}$-axis. Then, $A_{0}^{*}$, identified to a disc of $\mathbb{C}^{3}$, has two holomorphic lifts to $T^{*} \mathbb{C}^{3}$ attached to $T_{M_{0}}^{*} \mathbb{C}^{3}$. Their components are parametrized by $z_{1} \mapsto\left(0,-\frac{1}{z_{1}}, 1\right)$ and $z_{1} \mapsto\left(0, \frac{1}{i z_{1}}, \frac{1}{i}\right)$ respectively.

Proof. First, we notice that for any $z=\left(z_{1}, z_{2}\right) \in \mathbb{B}^{2}$ the disc $\tau \mapsto$ $\tau \frac{z}{\|z\|}$ is the only passing through $z$ and 0 . The lift attached to the 
projectivized conormal bundle of this disc is the constant $[\bar{z}]$. We have

$$
M_{0}=\left\{(z ;[\bar{z}]) z \in \mathbb{B}^{2} \backslash 0\right\} \cup\left\{(0 ;[\zeta]) \forall[\zeta] \in \mathbb{C P}_{1}\right\} .
$$

Clearly $M_{0}$ (or more precisely $M_{0}^{\text {reg }}$ ) has equation $r: z_{3}-\frac{\bar{z}_{2}}{\bar{z}_{1}}=0$. In particular the lift of $A_{0}$ to $\mathbb{P} T^{*} \mathbb{C}^{2}$ is $A_{0}^{*}(\tau)=((\tau, 0) ;[1,0])$ which in coordinates is expressed by $A_{0}^{*}(\tau)=(\tau, 0,0)$. Since $M_{0}$ is Levi flat, the space of holomorphic lifts contained in $T^{*} M_{0}$ has dimension two. For instance a basis for the space of lifts is given by

$\omega_{1}\left(z_{1}, z_{2}\right)=\partial \operatorname{Re} r=\left(\frac{z_{2}}{z_{1}^{2}},-\frac{1}{z_{1}}, 1\right)$ and $\omega_{2}\left(z_{1}, z_{2}\right)=\partial \operatorname{Im} r=\frac{1}{i}\left(-\frac{z_{2}}{z_{1}^{2}}, \frac{1}{z_{1}}, 1\right)$.

In particular, along $A_{0}^{*}$ the conormal bundle to $M_{0}$ is generated by $\omega_{1}\left(z_{1}, 0\right)=\left(0, \frac{-1}{z_{1}}, 1\right)$ and $\omega_{2}\left(z_{1}, 0\right)=\left(0, \frac{1}{i z_{1}}, \frac{1}{i}\right)$. As one can readily note both sections are holomorphic along $A_{0}^{*}$ and they are exactly the lifts of $A_{0}^{*}$ to the conormal bundle of $T_{M_{0}}^{*} \mathbb{C}^{3}$.

Remark 2.2. Note that if in the lemma above we consider the union of the lifts of discs passing through the point $P_{\zeta_{0}}=\left(\zeta_{0}, 0\right)$ the manifold resulting $M_{\zeta_{0}}$ still contains $A_{0}^{*}$ (i.e. the $z_{1}$ axis) and along the boundary of $A_{0}^{*}$ we have $\left.T M_{0}\right|_{\partial A_{0}^{*}}=\left.T M_{\zeta_{0}}\right|_{\partial A_{0}^{*}}$ and thus also $\left.T_{M_{0}}^{*} \mathbb{C}^{3}\right|_{\partial A_{0}^{*}}=$

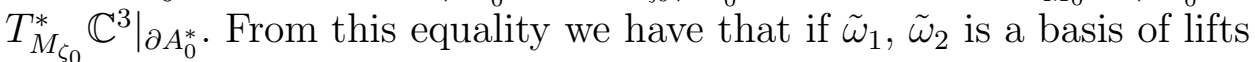
of $A_{0}^{*}$ to the conormal bundle to $M_{\zeta_{0}}$, then this is related to the basis $\omega_{1}, \omega_{2}$ by

$$
\left.\operatorname{Span}\left\{\tilde{\omega}_{1}, \tilde{\omega}_{2}\right\}\right|_{\partial A_{0}^{*}}=\left.\operatorname{Span}\left\{\omega_{1}, \omega_{2}\right\}\right|_{\partial A_{0}^{*}} .
$$

Combination of (2.2) with the fact that singularity of $\tilde{\omega}_{1}, \tilde{\omega}_{2}$ must now be located at $\zeta_{0}$ yields a choice of holomorphic basis as $\tilde{\omega}_{1}\left(z_{1}\right)=$ $\left(0,-\frac{1}{\left(z_{1}-\zeta_{0}\right)}, \frac{1}{\left(1-z_{1} \zeta_{0}\right)}\right)$ and $\tilde{\omega}_{2}\left(z_{1}\right)=\left(0, \frac{1}{i\left(z_{1}-\zeta_{0}\right)}, \frac{1}{i\left(1-z_{1} \zeta_{0}\right)}\right)$.

Before the proof of our main theorem we need a preliminary crucial result

Theorem 2.3. Let $P_{1}, P_{2} \in \mathbb{B}^{2}$ be two distinct points inside the ball and let $f: \partial \mathbb{B}^{2} \rightarrow \mathbb{C}$ be a continuous function such that $f$ extends holomorphically along every complex straight line passing through either $P_{1}$ or $P_{2}$. Then for any such disc $A$, except the one passing through both points, the lifted function $F$ extends holomorphically to a neighborhood of $A^{*} \backslash \pi^{-1}\left(P_{j}\right)$ where $j$ is 1 or 2 according to $P_{1} \in A$ or $P_{2} \in A$.

Proof. It is not restrictive to assume that the $\operatorname{disc} A$ is the $z_{1}$ axis, that $P_{2}=\left(0, z_{2}\right)$ and that $P_{1}=\left(\zeta_{0}, 0\right)$. We note that $M_{1}$ and $M_{2}$ intersect transversally along the boundary of $A^{*}$. Let $P=(\zeta, 0)$ be a point of 
the boundary of $A$ and $P^{*}=(\zeta, 0,0)$ be the corresponding point on $A^{*}$. $P^{*}$ lies in the common boundary of $M_{1}$ and $M_{2}$. Let $v_{\zeta}$ be a tangent vector to $T_{P^{*}} M_{2} \backslash T_{P^{*}} E$ which points inside $M_{2}$. The equivalence class $\left[v_{\zeta}\right]$ in the vector spaces quotient $\frac{T_{P^{*}} \mathbb{C}^{3}}{T_{P *} M_{1}}$ is called the pointing direction of $M_{2}$ with respect to $M_{1}$. We say in this case that $F$ extends at $P^{*}$ in direction $\left[v_{\zeta}\right]$. Let $Q^{*}=\left(\zeta_{Q}, 0,0\right)$ be a point of $A^{*}\left(\zeta_{Q} \neq \zeta_{0}\right)$. Following [13] by effect of the extension of $F$ at $P^{*}$ in direction $\left[v_{\zeta}\right]$ we have extension at $Q^{*}$ in direction $\left[w_{\zeta}\right] \in \frac{T_{Q^{*}} \mathbb{C}^{3}}{T_{Q^{*} M_{1}}}$. The relation of $\left[w_{\zeta}\right]$ with the initial $\left[v_{\zeta}\right]$ is expressed by means of contraction with the holomorphic basis of lifts $\tilde{\omega}_{1}, \tilde{\omega}_{2}$ :

$$
\operatorname{Re}\left\langle\tilde{\omega}_{1}(\zeta), v_{\zeta}\right\rangle=\operatorname{Re}\left\langle\tilde{\omega}_{1}\left(\zeta_{Q}\right), w_{\zeta}\right\rangle \text { and } \operatorname{Re}\left\langle\tilde{\omega}_{2}(\zeta), v_{\zeta}\right\rangle=\operatorname{Re}\left\langle\tilde{\omega}_{2}\left(\zeta_{Q}\right), w_{\zeta}\right\rangle \text {. }
$$

In other words the directions of $C R$ extendibility, which are vectors in the normal bundle $\frac{T \mathbb{C}^{3}}{T M_{1}}$, are constant in the system dual to $\left\{\tilde{\omega}_{1}, \tilde{\omega}_{2}\right\}$.

We first compute the pointing direction of $M_{2}$ at the point $P^{*}$. To this end we first compute the disc passing through $P_{2}$ and $P$ which is

$$
A_{P_{2} P}(\tau)=\left(\frac{\left|z_{2}\right|^{2} \zeta}{1+\left|z_{2}\right|^{2}}, \frac{z_{2}}{1+\left|z_{2}\right|^{2}}\right)+\frac{\tau}{1+\left|z_{2}\right|^{2}}\left(\zeta,-z_{2}\right)
$$

note that $A_{P_{2} P}(1)=P$. The lift component of $A_{P_{2} P}$ is

$$
A_{P_{2} P}^{*}=\left[\left|z_{2}\right|^{2} \bar{\zeta} \tau+\bar{\zeta}, \bar{z}_{2} \tau-\bar{z}_{2}\right]
$$

and dividing the second component by the first we get that the $A_{P_{2} P_{2}}^{*}$ 's coordinates in $\mathbb{C}^{3}$ are

$$
\left(\left(\frac{\left|z_{2}\right|^{2} \zeta}{1+\left|z_{2}\right|^{2}}+\frac{\tau}{1+\left|z_{2}\right|^{2}} \zeta, \frac{z_{2}}{1+\left|z_{2}\right|^{2}}-\frac{\tau z_{2}}{1+\left|z_{2}\right|^{2}}, \frac{\bar{z}_{2}(\tau-1)}{\bar{\zeta}\left(\left|z_{2}\right|^{2} \tau+1\right)}\right)\right.
$$

The pointing direction of $M_{2}$ at $P$ is

$$
v_{\zeta}=-\partial_{\tau} A_{P_{2} P}^{*}(1)=\frac{-1}{1+\left|z_{2}\right|^{2}}\left(\zeta,-z_{2}, \frac{\bar{z}_{2}}{\bar{\zeta}}\right) .
$$

We have

$$
\operatorname{Re}\left\langle\tilde{\omega}_{1}(\zeta), v_{\zeta}\right\rangle=\frac{-1}{1+\left|z_{2}\right|^{2}} \operatorname{Re} \frac{z_{2}}{\zeta-\zeta_{0}}
$$

and

$$
\operatorname{Re}\left\langle\tilde{\omega}_{2}(\zeta), v_{\zeta}\right\rangle=\frac{-1}{1+\left|z_{2}\right|^{2}} \operatorname{lm} \frac{z_{2}}{\zeta-\zeta_{0}}
$$

The first members of (2.4) and (2.5) express the components in the normal bundle to $M_{1}$ of $w_{\zeta}$ with respect to the dual basis of $\omega_{1}\left(\zeta_{Q}\right), \omega_{2}\left(\zeta_{Q}\right)$. By letting $\zeta$ vary in $\partial A$ we see that $\left[w_{\zeta}\right]$ sweeps all the directions in 
$\left.\frac{T \mathbb{C}^{3}}{T M_{1}}\right|_{Q^{*}}$. Therefore, by the edge of the wedge theorem, $F$ extends holomorphically to a neighborhood of $Q^{*}$ and, by propagation, to a neighborhood of any other point of $A^{*}$ except from the point over $P_{1}$ where the $C R$ singularity is located.

We are ready for the proof of Theorem 1.1 End of Proof of Theorem 1.1 Let $A_{0}$ be the disc passing through $P_{1}$ and $P_{3}$. Then in particular $P_{2} \notin A_{0}$. Applying the theorem above we get that $F$ extends holomorphically to a neighborhood of $A_{0}^{*} \backslash\left\{P_{1}\right\}$. By repeating the same argument we see that $F$ extends to a neighborhood of $A_{0}^{*} \backslash\left\{P_{3}\right\}$. Therefore $F$ extends to a full neighborhood of $A_{0}^{*}$. For any other straight line $A$ through $P_{1}$ we can say that $F$ extends holomorphically to a neighborhood of $A^{*} \backslash P_{1}$. By applying the continuity principle to the family of discs formed by $A_{0}^{*}$ and all the discs through $P_{1}$, we get that $F$ extends holomorphically to a set of the form $V \times \mathbb{C P}_{\mathbb{C}}^{1}$ where $V$ is a neighborhood of $P_{1}$. Therefore $F$ does not depend on the second argument and it is therefore naturally defined on the projection of the collection of all the $A^{*}$ s, that is, on $\mathbb{B}^{2}$.

\section{REFERENCES}

[1] M. Agranovsky - Holomorphic extension from the unit sphere in $\mathbb{C}^{n}$ into complex lines passing through a finite set math. arXiv 0910.3592 (2009)

[2] M. Agranovsky - Propagation of boundary CR foliations and Morera type theorems for manifolds with attached analytic discs. Adv. Math. 211 (2007), no. 1, 284-326.

[3] M. Agranovsky and A.M. Semenov-Boundary analogues of the Hartogs' theorem, Siberian Math. J., 32 (1991) Trans. A.M.S., 280 (1983), $247-254$

[4] M. Agranovky and R.E Valski-Maximality of invariant algebras of functions. (Russian) Sibirsk. Mat. Z. 12 (1971) 3-12.

[5] L. Baracco-Holomorphic extension from a convex hypersurface (2009)

[6] L. Baracco, A. Tumanov, G. Zampieri-Extremal discs and holomorphic extension from convex hypersurfaces. Ark. Mat. 45 (2007) 1-13

[7] J. Globevnik - Small families of complex lines for testing holomorphic extendibility Arxiv :0911.5088v2 (2009)

[8] J. Globevnik, E. L. Stout - Boundary Morera theorems for holomorphic functions of several complex variables. Duke Math. J. 64 (1991), no. 3, 571615

[9] L. Lempert - La metrique de Kobayashi et la representation des domaines sur la boule. Bull. Soc. Math. de France 109 (1981), 427-474

[10] W. Rudin - Function theory in the unit ball of Cn. Grundlehren der Mathematischen Wissenschaften [Fundamental Principles ofMathematical Science], 241. Springer-Verlag, New York- Berlin, (1980) 
[11] E. L. Stout - The boundary values of holomorphic functions of several complex variables. Duke Math. J. 44 (1977), no. 1, 105-108

[12] A. Tumanov Connections and propagations of analyticity for CR functions. Duke Math. J. 73 (1994), 1-24

[13] A. Tumanov Propagation of extendibility of $C R$ functions on manifolds with edges. Contemp. Math. 205 (1997),259-269

[14] A. Tumanov Testing analiticity on circles. Amer. J. Math. 129 (2007), no. $3,785-790$

[15] A. Tumanov-A Morera type theorem in the strip, Math. Res. Lett., 11 1 (204), 23-29

Dipartimento di Matematica, Università di Padova, via Trieste 63, 35121 PADOVA, ItALY

E-mail address: baracco@math.unipd.it 